PREPARED FOR THE U.S. DEPARTMENT OF ENERGY, UNDER CONTRACT DE-AC02-76CH03073

PPPL-3822

PPPL-3822

UC-70

Diagnostic Development for ST Plasmas on NSTX

by

D. Johnson and the NSTX Team

June 2003

N/M|

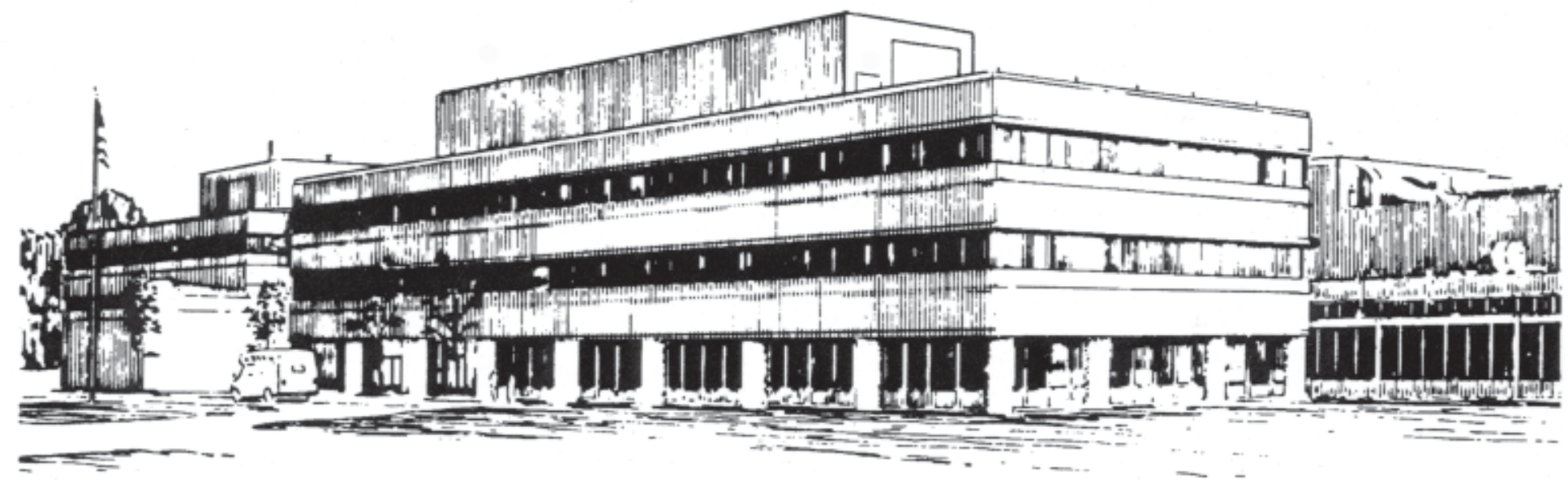

PRINCETON PLASMA PHYSICS LABORATORY PRINCETON UNIVERSITY, PRINCETON, NEW JERSEY 


\section{PPPL Reports Disclaimer}

This report was prepared as an account of work sponsored by an agency of the United States Government. Neither the United States Government nor any agency thereof, nor any of their employees, makes any warranty, express or implied, or assumes any legal liability or responsibility for the accuracy, completeness, or usefulness of any information, apparatus, product, or process disclosed, or represents that its use would not infringe privately owned rights. Reference herein to any specific commercial product, process, or service by trade name, trademark, manufacturer, or otherwise, does not necessarily constitute or imply its endorsement, recommendation, or favoring by the United States Government or any agency thereof. The views and opinions of authors expressed herein do not necessarily state or reflect those of the United States Government or any agency thereof.

\section{Availability}

This report is posted on the U.S. Department of Energy's Princeton Plasma Physics Laboratory Publications and Reports web site in Fiscal Year 2003. The home page for PPPL Reports and Publications is: http://www.pppl.gov/pub_report/

DOE and DOE Contractors can obtain copies of this report from:

U.S. Department of Energy

Office of Scientific and Technical Information

DOE Technical Information Services (DTIS)

P.O. Box 62

Oak Ridge, TN 37831

Telephone: (865) 576-8401

Fax: (865) 576-5728

Email: reports@adonis.osti.gov

This report is available to the general public from:

National Technical Information Service

U.S. Department of Commerce

5285 Port Royal Road

Springfield, VA 22161

Telephone: $1-800-553-6847$ or

(703) $605-6000$

Fax: (703) 321-8547

Internet: http://www.ntis.gov/ordering.htm 


\author{
Presented at \\ German-Polish EURO-Conference on \\ Plasma Diagnostics for Fusion and Applications \\ Greifswald, Germany \\ September 4-6, 2002
}

\title{
Diagnostic Development for ST Plasmas on NSTX*
}

\author{
D. Johnson and NSTX Team
}

\begin{abstract}
Spherical tokamaks (STs) have much lower aspect ratio $(\mathrm{a} / \mathrm{R})$ and lower toroidal magnetic field, relative to tokamaks and stellarators. This paper will highlight some of the challenges and opportunities these features pose in the diagnosis of ST plasmas on the National Spherical Torus Experiment (NSTX), and discuss some of the corresponding diagnostic development that is underway. The low aspect ratio necessitates a small center stack, with tight space constraints and large thermal excursions, complicating the design of magnetic sensors in this region. The toroidal magnetic field on NSTX is $\leq 0.6 \mathrm{~T}$, making it impossible to use ECE as a good monitor of electron temperature. A promising new development for diagnosing electron temperature is electron Bernstein wave (EBW) radiometry, which is currently being pursued on NSTX. A new high-resolution charge exchange recombination spectroscopy system is being installed. Since non-inductive current initiation and sustainment are top-level NSTX research goals, measurements of the current profile $J(R)$ are essential to many planned experiments. On NSTX several modifications are planned to adapt the MSE technique to lower field, and two novel MSE systems are being prototyped. Several high speed 2-D imaging techniques are being developed, for viewing both visible and x-ray emission. The toroidal field is comparable to the poloidal field at the outside plasma edge, producing a large field pitch $\left(>50^{\circ}\right)$ at the outer midplane. The large shear in pitch angle makes some fluctuation diagnostics like beam emission spectroscopy very difficult, while providing a means of achieving spatial localization for microwave scattering investigations of high-k turbulence, which are predicted to be virulent for NSTX plasmas. A brief description of several of these techniques will be given in the context of the current NSTX diagnostic set.
\end{abstract}

\section{Introduction}

NSTX device description - The National Spherical Torus Experiment (NSTX) is a national collaboration currently involving 21 US and 11 non-US institutions. It's goal is to produce and study high beta plasmas in a very low aspect ratio spherical tokamak (ST) device. NSTX has a major radius (R) of $0.85 \mathrm{~m}$, minor radius (a) of $0.68 \mathrm{~m}$, 
elongation $(\kappa)<2.5$ and aspect ratio, $\mathrm{R} / \mathrm{a} \geq 1.27$. The designed toroidal field $\left(\mathrm{B}_{\mathrm{T}}\right)$ of 0.6 $\mathrm{T}$, and a plasma current $\left(\mathrm{I}_{\mathrm{p}}\right)$ of 1.5 MA have been achieved. The plasma is heated by up to $7 \mathrm{MW}$ total of neutral beam injection (NBI) power from 3 co-injecting sources at 60 $110 \mathrm{keV}$ and up to $6 \mathrm{MW}$ of high harmonic fast wave (HHFW) at $30 \mathrm{MHz}(10-15$ times $\omega_{\mathrm{ci}}$ for hydrogen) coupled to the plasma through a 12-strap antenna. The center stack and inner divertor regions are electrically isolated from the outer vessel in order to permit coaxial helicity injection (CHI), which can provide up to $50 \mathrm{kA}$ of injector current. Thus far this current drive technique has produced a toroidal current of up to $400 \mathrm{kA}$. Wall stabilization is achieved with close fitting copper passive stabilizer plates. The plasma facing components (PFC's) which are subjected to plasma contact during high power operation are protected by a combination of graphite and carbon fiber composite tiles. Many of these features appear in the cutaway view shown in Fig. 1.

The PFC's are routinely baked to $350{ }^{\circ} \mathrm{C}$, while the outer vessel is maintained at $150{ }^{\circ} \mathrm{C}$. Between-shot $\mathrm{He}$ glow discharge cleaning and periodic surface boronization by a glow discharge in a mixture of deuterated trimethylboron gas and helium are utilized for wall conditioning.

Plasma performance - Since high power experiments began in 2000, plasma performance has improved steadily. Recently NSTX produced plasmas with a toroidal $\beta_{\mathrm{T}} \sim 34 \%\left(=2 \mu_{0}<\mathrm{p}>/ \mathrm{B}_{\mathrm{T}}\right.$ where $\mathrm{B}_{\mathrm{T}}$ is the vacuum field at the plasma geometric center $)$ and $\beta_{\mathrm{N}}\left(=\beta_{\mathrm{T}} \mathrm{aB}_{\mathrm{T}} / \mathrm{I}_{\mathrm{p}}\right) \sim 6 \% \mathrm{mT} / \mathrm{MA} . \mathrm{H}$-mode operation is now routine, with confinement times $\sim 1.5$ times ITER98pby2 scaling for several $\tau_{\mathrm{E}}$. In high power NBI experiments, $\mathrm{T}_{\mathrm{i}}(0) \sim$ $1.5-2.0 \mathrm{keV}$ and $\mathrm{T}_{\mathrm{e}}(0) \sim 1.0-1.5 \mathrm{keV}$ and central carbon rotation speeds up to $\mathrm{v}_{\mathrm{f}} / \mathrm{v}_{\text {Alfven }} \sim .33$ have been observed. In HHFW heating experiments, central $\mathrm{T}_{\mathrm{e}}(0)$ has 
reached nearly $4 \mathrm{keV}$. Transport analysis indicates that for most conditions ion confinement is very good, often near neoclassical predictions, while electron confinement is anomalous. This is consistent with microinstability theory, which indicates that, in many cases investigated, ITG mode growth rates are small and comparable to ExB shearing rates, while ETG growth rates are much larger.

General diagnostic considerations - From the point of view of diagnostics, ST's have several unique features to differentiate them from conventional tokamaks. While the weak toroidal field (TF) makes electron cyclotron emission techniques impossible and motional Stark effect polarimetry more difficult, the small physical size of the TF coils improves diagnostic access. Relative to tokamaks with similar $\mathrm{I}_{\mathrm{p}}$, weaker TF also means simplified design due to weaker eddy current forces. The ST geometry with the narrow center stack makes available wide angle views of nearly the entire plasma. The magnetic field pitch at the outer midplane on NSTX can be as high as $55^{\circ}$ and varies with the plasma current. This complicates reflectometry due to mode mixing, and limits the range and spatial resolution of techniques like beam emission spectroscopy and gas puff imaging, which rely on viewing fluctuations with sightlines parallel to the local field. On the other hand, the large field pitch helps to potentially improve the spatial resolution of microwave scattering techniques, as will be described later.

Table 1 shows the existing diagnostics on NSTX, as well as those currently being installed and those planned for installation in the next 1-2 years. The research program on NSTX is advancing very quickly, which motivates the development of fairly advanced turbulence diagnostics, even before more standard measurements are fully implemented. 
Indicated in square brackets [ ] in Table 1 are collaborating institutions responsible for fabricating and operating the diagnostics.

The remainder of this paper will discuss several ongoing diagnostic developments in various stages of implementation. This discussion will include a brief mention of the physics motivation for the measurement and the timetable for installation. It should be noted that, due to the diversity of NSTX research priorities and the variety of sources of funding for diagnostic development, there is also a considerable range of maturity of diagnostics supporting different research areas.

\section{Magnetics}

NSTX uses a conventional set of magnetic sensors, including 1-D and 2-D Mirnov coils, flux loops, Rogowski coils and a diamagnetic loop. The CS Mirnov coils were designed small enough to fit within cutouts in the $13 \mathrm{~mm}$ thick CS carbon tiles. These sensors also had to withstand the large thermal excursions (up to $600^{\circ} \mathrm{C}$ ) of the CS tiles, which are radiatively cooled. The CS Mirnov coils use bare copper wire wound on thin Macor mandrels.

A subset of the magnetic sensors are connected to a digital real time feedback system providing control of the plasma current, position and shape. The full set of signals is used in an equilibrium reconstruction performed between shots using the EFIT code. ${ }^{1}$ Recently a real-time reconstruction version (RTEFIT) has been demonstrated with a $10 \mathrm{~ms}$ time step. In the future Thomson and MSE profile data will also be input to this control system, with the goal of advanced profile control.

Significant improvements in the plasma performance occurred in the last run campaign following the realignment of a PF coil that produced a significant error field 
( 20 g) near the outer edge of the plasma. The misalignment was detected by a series of external 'picture frame' locked mode coils for measuring $\mathrm{B}_{\mathrm{r}}$. The realignment along with the introduction of $350^{\circ} \mathrm{C}$ bakeout reduced locked mode activity and improved access to H-modes. To improve sensitivity to residual unresolved field errors, a similar set of $B_{r}$ loops is being installed at 6 toroidal positions in grooves around groups of PFC tiles mounted on the upper and lower primary passive plates.

Resistive wall modes (RWM) have been observed on NSTX, with characteristic slowdown of plasma rotation. (see ref. 1) Although many of the high-beta discharges have been above the ideal no-wall beta limit, calculated by the DCON ideal stability code,$^{2}$ to extend this limit nearer to the ideal wall limit may require active stabilization of RWM modes. Hence at 6 toroidal positions on the midplane-facing edges of the primary passive plates, large $B_{p}$ sensor coils are being installed. Signals from these coils will be input to the digital plasma control system to control the currents in RWM stabilizer coils, which will be installed in 2003 .

\section{Profile Diagnostics}

EBW radiometry - At the low toroidal field and moderate densities on NSTX, $\Omega_{\mathrm{ce}}<<\omega_{\mathrm{pe}}$, and electromagnetic radiation cannot progagate for the first several cyclotron harmonics. Radiation at higher harmonics will propagate but does not satisfy the blackbody condition necessary for a monitor of electron temperature. Electron Bernstein waves (EBW's) are electrostatic waves that can propagate in ST plasmas and exhibit high optical thickness at the electron cyclotron resonances. These waves can only exist within the upper hybrid resonance layer typically located near the outside edge of the plasma. If the density scale length $L_{n}{ }^{U H R}$ at this layer is short enough, these waves will mode-convert 
and tunnel to fast X-mode electromagnetic waves, which can be easily detected and provide information on $T_{e}(R, t)$.

Studies are underway at PPPL to measure the mode conversion efficiency and its strong dependence on $\mathrm{L}_{\mathrm{n}}{ }^{\mathrm{UHR}}{ }^{3}$. On NSTX, conversion efficiencies as high as $40 \%$ have been observed, in agreement with model calculations ${ }^{4}$ based on measured $\mathrm{L}_{\mathrm{n}}^{\mathrm{UHR}}$. On CDX-U, a local, radially-scannable limiter has been used to modify $\mathrm{L}_{n}{ }^{\mathrm{UHR}}$ in the vicinity of the receiving horn, and thereby maximize the conversion efficiency at near $100 \%$, based on comparisons with core Thomson temperatures. On NSTX, a similar receiving horn/limiter assembly is being installed, shown schematically in Fig. 2. Two radially moveable graphite limiters, oriented normal to the local field on either side of the receiver, will be used to locally modify $L_{n}{ }^{U H R}$. A second microwave horn will be used in reflectometer mode to monitor the local density profile evolution. Results from these studies are important, not only for demonstrating the feasibility of EBW radiometry for fast $T_{e}(R, t)$ measurements, but also for gaining confidence in high power EBW for heating and current drive of ST plasmas.

Thomson scattering - Since ECE measurements are not possible at the low magnetic field on NSTX, and EBW radiometry is under development, Thomson scattering provides critical $T_{e}(R, t)$ profiles as well as $n_{e}(R, t)$ profiles. The NSTX Thomson scattering diagnostic is a conventional Nd:YAG system with a midplane, backscattering geometry designed for high spatial resolution and high sensitivity at the outer edge of the plasma. ${ }^{5}$ At the outer separatrix, the spatial resolution is $\sim 0.6 \mathrm{~cm}$ and light is collected at $\sim \mathrm{f} / 3.5$. The system is modular and not yet fully implemented. It is capable of a full midplane profile with $\sim 40$ spatial positions, with 20 channels currently 
instrumented with polychromators and detectors. Two Spectra Physics $30 \mathrm{~Hz}, 1.6 \mathrm{~J}$ lasers are now in use, with space for a third. The lasers feature feedback-stabilized aiming, and realignments during 4-5 month run campaigns have not been necessary. The system uses the General Atomics polychromator design. ${ }^{6}$ Custom low noise preamps and sample-and-hold electronics were developed for this system. ${ }^{7}$ A series of absolute calibrations are confirmed by agreement with FIR interferometry for line density measurements to $\pm 5 \%$. As previously mentioned, raw data from this system will be input to the digital control system in the near future. Plasma background data from a narrow spectral channel has also been used to derive $\mathrm{Z}_{\text {eff }}$ profiles. ${ }^{8}$

Charge exchange recombination spectroscopy - A charge exchange recombination spectroscopy diagnostic (CHERS) provides profiles measurements of impurity carbon $\mathrm{T}_{\mathrm{i}}$ and $\mathrm{v}_{\phi}$. Central ion temperatures are typically $1-2 \mathrm{keV}$, and toroidal rotation speeds up to $280 \mathrm{~km} / \mathrm{sec}$ have been measured. The diagnostic is currently being upgraded from a 17 to a 51 channel system, optimized for high resolution at the outer separatrix. The system uses the heating beam with an optimized tangential view that is shared with the MSE/CIF diagnostic (see below), as shown in Fig. 3. Resolution of the new system ranges from $\sim 0.5 \mathrm{~cm}$ at the edge (comparable to ion gyroradius and well matched to Thomson) to $\sim 3.0 \mathrm{~cm}$ in the core. The diagnostic utilizes the $\mathrm{C}^{5+}(\mathrm{n}=8-7)$ line at $529.1 \mathrm{~nm}$ emitted by ions which are excited by charge exchange collisions with injected $80 \mathrm{keV} \mathrm{D}{ }^{0}$ beam atoms. A background view (which does not see the beam) and a dedicated spectrometer/detector system are used to separately measure the cold background component of the line, typically comparable in brightness, arising from the intrinsic emission shell near the plasma edge. The imaging spectrometers are high 
throughput f/1.8 Kaiser Holospec instruments and the detectors are Princeton Instruments Pentamax Cameras operated with $10 \mathrm{~ms}$ frame time, similar to those used in the TFTR poloidal rotation diagnostic. ${ }^{9}$ To make optimum use of each spectrometer/detector, we use interference filters to isolate spectra from multiple slits, permitting many spectra to be recorded simultaneously with one spectrometer: 51 spectra on primary spectrometer and 39 spectra on background spectrometer. This configuration is shown in Fig. 4. Accurate background subtraction requires careful white-plate and spectral calibrations for both views. A Ne glow discharge in NSTX has been used to calibrate the instrumental widths for each channel, using the NeI lines at 529.819 and $530.476 \mathrm{~nm}$.

Edge Doppler spectroscopy - A nearly identical spectrometer/detector configuration is now being installed to measure the rotation at the plasma edge. The diagnostic measures Doppler shifts in intrinsic $\mathrm{C}^{2+}(465 \mathrm{~nm})$ and $\mathrm{C}^{3+}(468 \mathrm{~nm})$ lines, covering (with down to $\sim 1 \mathrm{~cm}$ spacing) a radial region of $15 \mathrm{~cm}$ at the extreme edge of the outboard midplane. Thirteen chordal views are distributed between toroidal and poloidal sightlines, allowing the toroidal and poloidal rotation of the plasma edge to be characterized with $10 \mathrm{~ms}$ resolution. Combined with the local pressure gradient and magnetic field profile, the edge flow will give a measure of the local radial electric field.

Motional Stark Effect Polarimetry - One of the major NSTX performance goals is the attainment of high $\beta$ ( $\beta \sim 40 \%)$, long pulse discharges with high noninductive current fraction (bootstrap fraction $\sim 0.7-0.8$ ). Non-inductive current fractions of $\sim 0.5$ have already been inferred from modeling for some discharges. Measurement of $j(R)$ is critical and considerable effort has been made, in collaboration 
with NOVA Photonics, to adapt motional Stark effect polarimetry (MSE) to provide this capability on NSTX.

The first of two planned MSE diagnostics is currently being installed on NSTX. ${ }^{10}$ This system will use emission from collisionally-induced-fluorescence (CIF) arising from collisions between injected $\mathrm{D}^{0}$ heating beam atoms and the background plasma. Several modifications have been made to improve isolation of the Stark polarization components, whose spectral splitting is reduced at the low B fields of the ST. First, the viewing aperture has been narrowed toroidally, thereby reducing the Doppler spreading of individual components due to the spread in viewing angles. To compensate, the aperture is elongated vertically. Second, a high throughput, narrowband birefringent Lyot filter has been developed with a bandpass of $0.075 \mathrm{~nm}$ FWHM, compared to the $0.7 \mathrm{~nm}$ bandwidth attainable from a standard interference filter. Fig. 4 shows a photo of the 3stage filter. These filters must be temperature stabilized to $\leq 0.01^{\circ} \mathrm{C}$. The crystals have conductive coatings which permit electronic tuning.

Even with these modifications, the polarization fraction of the detected light is predicted to be in the range $0.2-0.4$, much smaller than the value of 0.8 typical for this technique on high field devices. To compensate for the resulting loss in $\mathrm{S} / \mathrm{N}$, the optical throughput is increased for the NSTX system. Each of the 19 spatial channels receives light from $761 \mathrm{~mm}$ fibers, which is imaged with aspheric optics through the Lyot filters and onto a $1.0 \mathrm{~cm}$ diameter avalanche photodiode. Spatial resolution will be $2-3 \mathrm{~cm}$, with coverage from $10-20 \mathrm{~cm}$ inboard of the magnetic axis to the outer edge. The collection optics, photoelastic modulators, and fiber optics were recently installed on NSTX, and two prototype filter/detector systems are being assembled and tested. The 
goal is to instrument 10 spatial channels and produce pitch angle profile measurements by mid-'03.

On a longer development path, a second MSE system is planned for NSTX with installation scheduled for ' 05 . This system will use a compact $\mathrm{H}^{0}$ beam with a coaxial tunable ring dye laser resonant with the Doppler shifted $\mathrm{H}_{\alpha}$ transition for the beam atoms. The linear polarization of the laser is rotated and the phase between this rotation and the intensity of the laser-induced-fluorescence (LIF) is used to determine the pitch angle. This system is currently being prototyped in the laboratory. ${ }^{11}$ For this system to be feasible with practical laser energies, the $\mathrm{H}^{0}$ beam needs to very mono-energetic, with parallel energy spread of a few volts. The current effort is aimed at reducing ripple and RF noise in the beam HV power supply and at improving the RF ion source to further reduce the energy spread and to improve the atomic fraction of the beam. On NSTX the

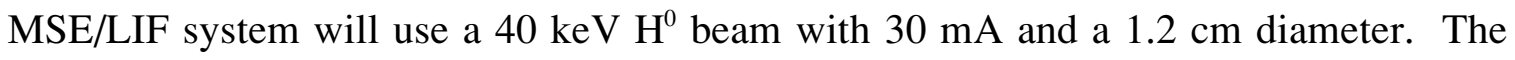
laser is an argon ion pumped ring dye laser with a CW output of 0.6 watts, which can be upgraded to 2-3 watts. A high throughput optical system will image the LIF onto $~ 20$ channels covering the outer half of the plasma with $2-3 \mathrm{~cm}$ resolution. The DNB for the MSE/LIF system will be injected radially on NSTX to eliminate $E_{r}$ effects in the determination of the pitch angle. In principle, therefore, data from both MSE systems on NSTX will permit determination of $E_{r}(R)$ as well as $j(R)$.

FIR laser tagential interferometer/polarimeter - In collaboration with UC Davis, NSTX is developing a FIR laser tangential interferometer/polarimeter ${ }^{12,13}$ (FIReTIP), for fast measurements of electron density and toroidal field. This system currently has 4 channels instrumented ( 7 planned) in a midplane tangential fan array. 
The system is based on the three-laser technique first used by Rommers and Howard on RTP. ${ }^{14}$ Two slightly detuned FIR lasers $\left(\mathrm{CO}_{2}\right.$ pumped $\mathrm{CH}_{3} \mathrm{OH}$ lasers at $\left.119 \mu\right)$ are combined to form right and left hand circularly polarized probing beams. The line density is derived from the average phase shift induced by the plasma in these beams, while the phase difference will be twice the Faraday rotation angle. A third FIR laser, which is Stark-tuned on NSTX, is operated at a difference frequency of $\sim 5 \mathrm{MHz}$, and is used as a local oscillator. ${ }^{15}$ The large difference frequency permits high frequency response ( $>100 \mathrm{kHz}$ ) for the system with good immunity to fringe jumps. Ex-vessel retro-reflectors are used, which are mechanically isolated from the NSTX device. This system is not vibration compensated, although vibration isolation is used on the common optical table and on the individual retro-reflector mounts.

In an ST at high $\beta$, the toroidal field can deviate significantly from vacuum values, and careful measurements of the Faraday rotation and line density along the chords of the FIReTIP system can, in principle, provide valuable constraints to the equilibrium reconstructions. Simulations indicate that the deviation of from vacuum values $\left(\Delta \mathrm{B}_{\mathrm{TF}} \sim 10^{2} \mathrm{~g}\right)$ produces several degrees of Faraday rotation angle, while expected precision for an optimized system is $\sim 0.05^{\circ}$. Effort on the FIReTIP system has initially been on interferometry and providing real-time density signals to the digital control system, although initial Faraday rotation data has been obtained, and noise sources are being eliminated.

SOL and edge $n_{e}$ profile reflectometer systems - NSTX utilizes a number of reflectometer systems to measure the SOL and edge density profiles, to measure MHD and fluctuations, and more recently to probe the edge magnetic field. The SOL $n_{e}$ profile 
system, developed by ORNL, is installed between two straps of the HHFW antenna. With a frequency range of $6-36 \mathrm{GHz}$, it is designed to cover the density range 0.02 to $1.6 \times 10^{19} \mathrm{~m}^{-3}$ with a sweep time that can be varied from $100-850 \mu \mathrm{sec}$. This system has been useful in the optimization of RF coupling for the HHFW heating system. UCLA has developed a second set of reflectometers with 3 bands in the range $12-50 \mathrm{GHz}{ }^{1617}$ Profile measurements via frequency-modulated, continuous-wave (FMCW) operation are typically made in O-mode polarization, with sweep times of $100 \mu \mathrm{sec}$. This system has recently measured fast changes in edge density scale length $L_{n} \Rightarrow 15$ to $2 \mathrm{~cm}$, over $1-2 \mathrm{~ms}$ during H-mode transitions and ELMs. Operated in fixed- or stepped-frequency mode, the UCLA system has been useful in detected the presence of fluctuations, for example, providing independent measurements of the frequency spectrum of CAE modes. Both of these reflectometer systems employ circular waveguides and polarization rotators so that the wave polarization can be adjusted relative to direction of the edge magnetic field.

Recently, UCLA has also been exploring techniques to measure the magnetic field in the edge region of NSTX. One experiment followed on work done at LAPD ${ }^{18}$ and was designed to measure $|\mathrm{B}|$ by launching $\mathrm{O}$ - and $\mathrm{X}$-mode radiation from the same antenna, holding one frequency fixed while sweeping the other. The peak in the crosscorrelation of fluctuating signals from naturally occurring turbulence can then be used to derive $|\mathrm{B}|$. Correlations were sharp enough to yield measurement precision of $0.5-1.0 \%$, and values were consistent with those derived from EFIT reconstructions. UCLA has also proposed measuring the dynamics of the edge magnetic field pitch by correlating fluctuation signals from an array of reflectometers positioned to span a range of pitch angles in the edge region. This concept makes use of the evidence that turbulent 
streamers are aligned to the field $\left(\mathrm{k}_{\|} / \mathrm{k}_{\perp}<<1\right)$. Accuracy of $<2^{\circ}$ with time resolution of $\sim$ $1 \mathrm{~ms}$ appear feasible.

\section{Core Fluctuation diagnostics}

Micro-instability analysis of NSTX neutral beam heated discharges using GS2 indicate that high-k ETG modes are unstable over much of the minor radius, while low-k ITG modes have much lower growth rates and are likely to be stabilized by flow shear. ${ }^{19}$ This is consistent with energy transport analysis based on kinetic profiles, which indicates that ions are well confined at or near neoclassical values, whereas the electron channel appears very lossy. Fluctuation diagnostics able to probe for both high and low k fluctuations are being considered for NSTX.

Imaging reflectometry - NSTX is planning, over the next 1-2 years, to implement an imaging reflectometer, similar to that being developed by FOM/UC Davis/ PPPL on TEXTOR. ${ }^{20}$ As demonstrated recently by careful laboratory characterization ${ }^{21}$, this new approach holds considerable promise for extending the applicability of reflectometer fluctuation measurements. One of the difficulties in implementing this diagnostic is the large optical access needed, which, on NSTX is aided by the fact that the viewing window can be quite close $(\sim 40 \mathrm{~cm})$ to the plasma region of interest. Fig. 5 shows the proposed optical design for this NSTX system.

$1 \mathrm{~mm}$ microwave scattering - Because of their small scale, for ETG modes to affect plasma transport, radially elongated eddies should exist. For initial probing for this turbulence, Mazzucato has proposed ${ }^{22,23}$ a $1 \mathrm{~mm}$ microwave scattering configuration where the beam is launched tangent to a magnetic surface from a point on the midplane. Scattering occurs in a region with a radial extent of $\sim 4 \mathrm{~cm}$ near the tangency point. In 
this initial experiment, scattered beams are collected at 3 discrete angles corresponding to available midplane windows and to $\mathrm{k}_{\mathrm{r}} \sim 8,20$, and $30 \mathrm{~cm}^{-1}$, a spectral range where ETG modes are predicted on NSTX.

If significant turbulence is observed at high-k in this initial investigation, more extensive spectral and spatial information could be obtained with another scattering geometry, with more complicated collection optics, which makes use of the large shear in the magnetic field pitch on NSTX. A $1 \mathrm{~mm}$ microwave beam with a waist of $2 \mathrm{~cm}$ is launched through a window $\sim 30 \mathrm{~cm}$ above the midplane and traverses the plasma nearly radially past the narrow center stack to a scattering region in the outboard side of the plasma (since the turbulence is predicted to be much stronger on the outboard side). Using a large in-vessel mirror, the scattered light is imaged through a window to a detector plane, where light from different values of $\mathrm{R}$ and $\mathrm{k}_{\perp}$ is dispersed in 2 dimensions. Radial resolution of $1-3 \mathrm{~cm}$ for the outer $90 \%$ of the plasma, and spectral resolution of $\sim 1 \mathrm{~cm}^{-1}$ for $\mathrm{k}_{\perp} \sim 10-40 \mathrm{~cm}^{-1}$ appear feasible. Dispersion adequate for this resolution is only possible because of the large pitch angle shear in the ST equilibrium. (This was pointed out first for tokamaks by P. Devynck, et $\mathrm{al}^{24}$ ) Using available sources and detectors, such a system is predicted to easily have adequate sensitivity to detect the fluctuation amplitudes $\left(\delta \mathrm{n} / \mathrm{n} \sim 10^{-3}\right)$ predicted from a mixing length criterion for NSTX parameters.

\section{Visible and $X$-ray Fast Imaging}

Gas puff imaging - A new diagnostic technique has been developed on NSTX and C-Mod to study edge turbulence. ${ }^{25}$ A gas puff of $\mathrm{He}$ or D is used to illuminate turbulent filaments, which are aligned to the magnetic field. On NSTX the puff 
originates at a $30 \mathrm{~cm}$ long manifold with many orifices along its length located $3-5 \mathrm{~cm}$ from the separatrix and oriented normal to the local field direction. An optical system views the puff such that the sightlines are parallel to the magnetic field at the puff location. A special CCD camera has been developed for this purpose by Princeton Scientific Instruments. ${ }^{26}$ In this PSI-IV camera, each of the 160x80 "superpixels" (115 $\mu$ pitch) has embedded storage pixels for 28 exposures. The optical filling factor is $\sim 0.5$. The camera runs continuously overwriting the storage pixels at rates up to $10^{6} \mathrm{f} / \mathrm{sec}$ until a readout trigger is received, when the stored frames are slowly read. Readout noise is 10e with a maximum signal of $\sim 30000$ e. On NSTX, the radial resolution of the full system is $\sim 3 \mathrm{~cm}$ due to the large parallel extent of the gas cloud, and the poloidal resolution is $\sim 3 \mathrm{~mm}$. The resulting movies (using HeI light at $587.6 \mathrm{~nm}$ ) show interesting edge turbulence dynamics with "blobs" and waves with characteristic scale length of 5-10 $\mathrm{cm}$ and velocities of $\sim 1 \mathrm{~m} / \mathrm{ms}$ in L-mode plasmas, with clear reductions in turbulence in $\mathrm{H}$-mode.

For detailed studies of disruption and reconnection dynamics, magnetic islands, and MHD mode structure, there is interest in using 2-D tangential $\mathrm{x}$-ray cameras on NSTX, and there has been considerable development in this area.

Tangential GEM camera - Recently, a tangential pinhole camera utilizing a 12 x 12 pixel Micro Pattern Gas Detector (MPGD) having a Gas Electron Multiplier (GEM) as amplifying stage was installed, in collaboration with ENEA Frascati (Italy) and Johns Hopkins University. ${ }^{27}$ Since each pixel is a proportional counter, GEM gain and discriminator thresholds can be used to select the x-ray energy range. Fast, low noise electronics permit single photon count rates to $10 \mathrm{e} 7 \mathrm{ph} / \mathrm{s} / \mathrm{pixel}$ and framing rates to 100 
$\mathrm{kHz}$. The $2.5 \mathrm{~cm} \times 2.5 \mathrm{~cm}$ detector is easily moved behind the pinhole to change the location and size of the probed region. Recent results on NSTX were restricted, because of the $12 \mu$ Be vacuum window, to energies $2.5 \mathrm{keV} \leq \mathrm{E}_{\mathrm{h} v} \leq 10 \mathrm{keV}$ and frame rates $\leq 50$ $\mathrm{kHz}$ for reasonable $\mathrm{S} / \mathrm{N}$. However, it is straightforward to utilize thinner windows, and hence greatly increase the signals to achieve higher frame rates. It also appears straightforward to extend this technology to higher pixel count cameras.

2-D burst-mode tangential x-ray camera - Princeton Scientific Instruments, in collaboration with PPPL, is developing a tangential x-ray camera utilizing one of their ultra-fast CCD cameras as a readout device. Similar to systems developed for TEXTOR and LHD $^{28}$, this system (shown in Fig. 6) features a wide-angle tangential view, variable pinholes and foils, a phosphor-coated faceplate vacuum interface, and a gated image intensifier tube with a fast phosphor. The electrostatic image tube demagnifies the 80 $\mathrm{mm}$ input diameter to match the $13 \times 13 \mathrm{~mm}$ CCD size at the $\mathrm{P} 46$ phosphor anode. The output image is lens-coupled to the CCD. The camera is a PSI-V CCD with $64 \times 64$ pixels and 300 frame storage capability with frame rates up to $500 \mathrm{kHz}$. The sensor chip architecture is similar to that for the PSI-IV camera described above. The image tube gain is adjusted to provide $\sim 1$ electron-hole pair in the CCD per x-ray photon. The detector is currently being calibrated with an x-ray source on the bench, and is scheduled for installation on NSTX in $\sim 6$ months. This system requires significant magnetic and radiation shielding.

2-D continuous fast tangential camera - Johns Hopkins University has proposed a novel concept for a continuous high-speed x-ray camera utilizing ASIC chip technology for data acquisition. This concept utilizes a high efficiency $\mathrm{CsI}(\mathrm{Tl})$ scintillator behind a 
pinhole. Converted light is transported by high efficiency light guides to a series of multi-anode (8x8) PMTs or APD arrays. Outputs are connected to 128 channel ASIC data acquisition boards featuring simultaneous charge integration, digitization, and readout. These boards, developed by Electron Tubes (XDAS-01) for x-ray scanning of baggage, are currently capable of $10 \mathrm{kHz}$ readout, but modifications for $100 \mathrm{kHz}$ continuous operation appear feasible.

\section{Fast Lost Ion Probe}

Fast ion loss on NSTX is predicted to increase with $\beta$ and can be significant at low current. (Loss fraction is calculated to be $\sim 40 \%$ for $\beta_{\mathrm{T}}=40 \%$ and $\mathrm{I}_{\mathrm{p}}=1.0 \mathrm{MA}$.) To investigate this loss, a scintillator-based fast lost ion probe (FLIP) is being installed on NSTX, similar to those developed on TFTR. ${ }^{29}$ This probe features an aperture located $2 \mathrm{~cm}$ behind the shadow of the RF limiter. Fast ions on loss orbits, which pass through the aperture, hit a scintillator at a position which depends on their gyroradius and pitch angle. The emitted light is imaged through a vacuum window onto a coherent fiber bundle and sensed with an intensified CCD camera. A novel feature of this probe is the array of 10 aluminum Faraday collectors deposited between the scintillator and the substrate, and shaped to correspond to intervals of pitch angle. These Faraday collectors will provide higher time resolution and a simple means of calibration. A heavy copper support acts as a heat sink to keep the P46 phosphor temperature $<200^{\circ}$ C. Because of the low field and large gyroorbits on NSTX, the probe size is $\sim 8$ times the linear dimension of the TFTR probes. 


\section{Summary}

This paper reports on the status of the broad range of diagnostic development, which is needed to keep pace with the rapid research progress on NSTX. Many of these developments are in response to challenges and opportunities specific to the ST configuration. The NSTX program is fortunate to have a large team of diagnostic experts from many institutions contributing to this effort. The ST program goals involve extensions of physics parameters to provide insight by expanding the boundaries of the toroidal confinement database. Similarly, it is hoped that diagnostic techniques developed in this effort will benefit the broader community.

* Work supported by U. S. DOE Contract DE-AC02-76CH03073

\section{References}

${ }^{1}$ S. A. Sabbagh, et al., Nucl. Fusion, 41, 1601 (2001)

${ }^{2}$ A. H. Glasser, et al., Bull. Am. Phys. Soc. 42, 1848 (1997).

${ }^{3}$ G. Taylor, et al., Proc. $14^{\text {th }}$ Topical RF Conference, AIP Conf. Proc. $<595>, 282$ (AIP, New York 2001)

${ }^{4}$ A.K. Ram and S.D. Schultz, Phys of Plasmas 7, 4084 (2000).

${ }^{5}$ B. LeBlanc, et al., paper BP38 presented at the $14^{\text {th }}$ HTPD, Madison, Wisconsin, July 2002, to be published in Rev. Sci. Instrum.

${ }^{6}$ T. N. Carlstrom, et al., Rev. Sci. Instrum., 63, 4901 (1992)

${ }^{7}$ D.W. Johnson, et al., Rev. Sci. Instrum., 70, 1129 (1999)

${ }^{8}$ J. Menard, et.al., Nuclear Fusion, 41, 1197, (2001)

${ }^{9}$ R. E. Bell, Rev. Sci. Instrum., 70, 821 (1999)

${ }^{10} \mathrm{~F}$. Levinton, et al., paper BP16 presented at the $14^{\text {th }}$ HTPD, Madison, Wisconsin, July 2002, to be published in Rev. Sci. Instrum.

${ }^{11} \mathrm{~J}$. Foley, et al., paper BP15 presented at the $14^{\text {th }}$ HTPD, Madison, Wisconsin, July 2002, to be published in Rev. Sci. Instrum.

${ }^{12}$ H. Park, et al., Rev. Sci. Instrum. 70, 710 (1999)

${ }^{13}$ B. H. Deng, et al., paper BP23 presented at the $14^{\text {th }}$ HTPD, Madison, Wisconsin, July 2002, to be published in Rev. Sci. Instrum.

${ }^{14}$ J. H. Rommers, et al., Plasma Phys. Control. Fusion, 38, 1805 (1996)

${ }^{15} \mathrm{~K}$. C. Lee, et al., paper BP24 presented at the $14^{\text {th }}$ HTPD, Madison, Wisconsin, July 2002, to be published in Rev. Sci. Instrum.

${ }^{16}$ S. Kubota, et al., Rev. Sci. Instrum., 72, 348 (2001) 
${ }^{17} \mathrm{~S}$. Kubota, et al., paper AP13 presented at the $14^{\text {th }}$ HTPD, Madison, Wisconsin, July 2002, to be published in Rev. Sci. Instrum.

${ }^{18}$ M. Gilmore, et al., Rev. Sci. Instrum., 72, 293 (2001)

${ }^{19}$ C. Bourdelle, et al., Joint US-EU TTF Mtg. Fairbanks, Alaska, May 16, 2001

${ }^{20}$ A.J.H. Donné, et al., these proceedings

${ }^{21}$ T. Munsat, et al., paper AI04 presented at the $14^{\text {th }}$ HTPD, Madison, Wisconsin, July 2002, to be published in Rev. Sci. Instrum.

${ }^{22}$ E. Mazzucato, proposal submitted to US Department of Energy Office of Science Notice 01-25, August, 2001.

${ }^{23} \mathrm{H}$. Park, et al., paper AP08 presented at the $14^{\text {th }}$ HTPD, Madison, Wisconsin, July 2002, to be published in Rev. Sci. Instrum.

${ }^{24}$ P. Devynck, et al., Plasma Physics and Control. Fusion, 35, 63 (1993)

${ }^{25}$ R. Maqueda, , et al., paper FI02 presented at the $14^{\text {th }}$ HTPD, Madison, Wisconsin, July 2002, to be published in Rev. Sci. Instrum.

${ }^{26}$ Princeton Scientific Instruments web site: www.prinsci.com

${ }^{27}$ D. Pacella, et al., paper GP03 presented at the $14^{\text {th }}$ HTPD, Madison, Wisconsin, July 2002, to be published in Rev. Sci. Instrum.

${ }^{28}$ S. vonGoeler, et al., Rev. Sci. Instrum. 70, 599 (1999)

${ }^{29}$ D. Darrow, et al., Rev. Sci. Instrum. 66, 476 (1995) 


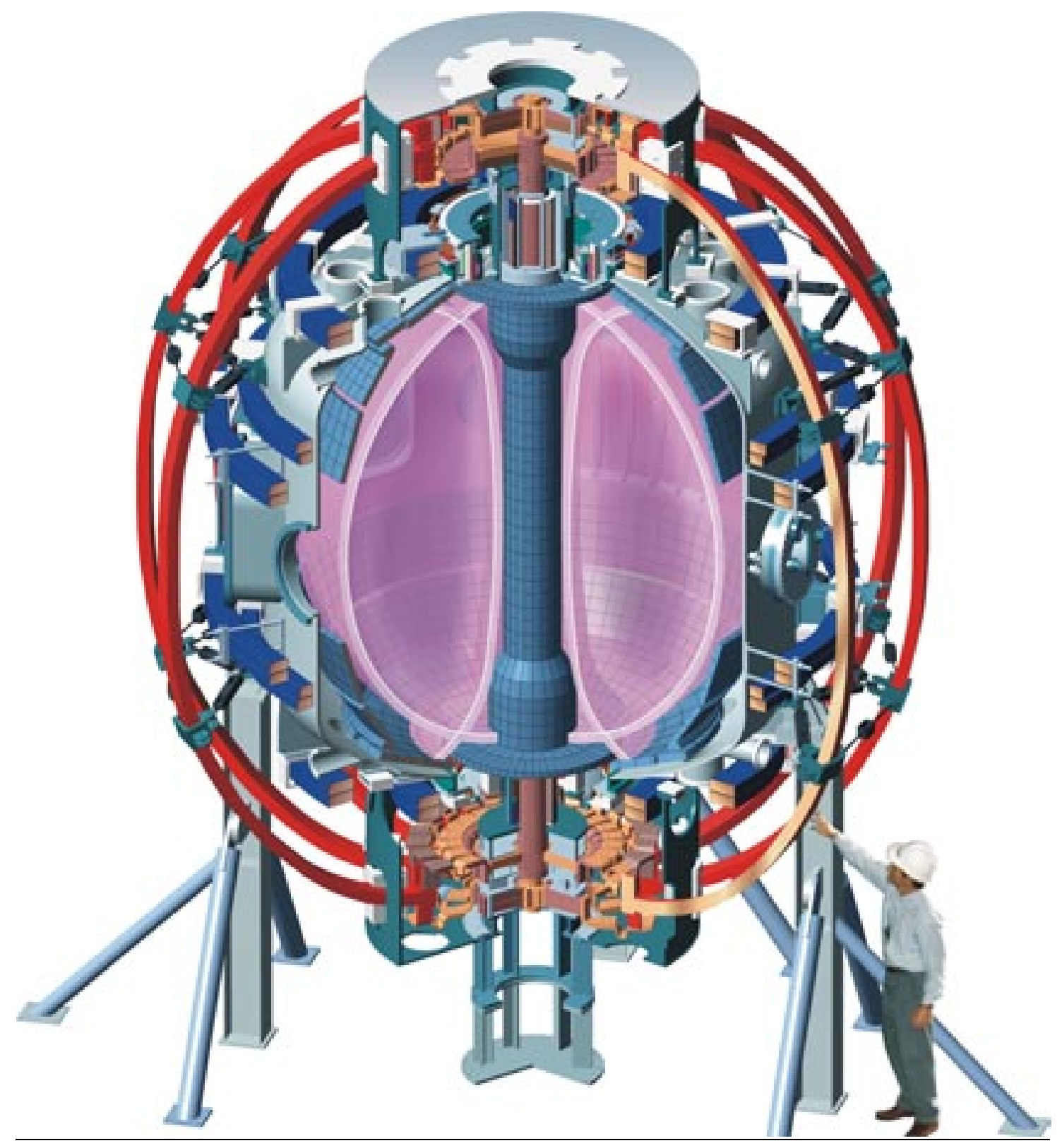

Figure 1. A cutaway view of the NSTX device showing in-vessel features including removable, narrow center stack, top and bottom divertor regions, outboard passive stabilizer plates, and external features including small TF coils and large midplane ports close to the plasma. 


\section{Confinement Studies}

- Magnetics for equilibrium reconstruction

- Diamagnetic flux measurement

- Thomson scattering $(20$ ch., $60 \mathrm{~Hz})$

- $1 \mathrm{~mm}$ interferometer/polarimeter [UCLA]

- FIReTIP $119 \mu \mathrm{m}$ tang. interf/polar.(4 -7 ch*) [UCD]

- Imaging X-ray crystal spectrum. $\left(\mathrm{T}_{\mathrm{i}}(0), \mathrm{T}_{\mathrm{e}}(0)\right)$

- CHERS $\left(\mathrm{T}_{\mathrm{i}} \& \mathrm{v}_{\phi}\right)\left(16-51 \mathrm{ch}^{*}\right)$

- Edge Doppler spectroscopy: $\mathrm{v}_{\mathrm{pol}}{ }^{*}$

- Electron Bernstein wave radiometer

- MSE/CIF polarimeter [NOVA]*

- $\mathrm{MSE} / \mathrm{LIF}$ polarimeter [NOVA] ${ }^{+}$

\section{MHD/Fluctuations}

- Locked mode coils

- High-n and high-frequency Mirnov arrays

- Soft x-ray arrays (3-5) [JHU]

- Edge reflectometer [UCLA]

- Edge fluctuation imaging [LANL,PSI]

- GEM tangential x-ray camera [JHU,ENEA]

- Ultra-fast tangential x-ray camera[PSI]*

- RWM/error field sensors*

- Scintillator-based X-ray pinhole camera[JHU $]^{+}$

- $1 \mathrm{~mm}$ microwave scattering ${ }^{+}$

- Imaging reflectometer ${ }^{+}$

\section{Plasma Monitoring}

- Fast visible cameras [LANL]

- VIPS-1, VIPS-2:Visible spectrometers

- VB detector (single chord)

- SPRED: UV spectrometer (CCD)

- GRITS: VUV spectrometer [JHU]

- Trans. grating imaging spect. [JHU]

- 1-D CCD Hameras [ORNL]

- Visible filterscopes $\left(\mathrm{H}_{\mathrm{a}}, \mathrm{OII}, \mathrm{CII}\right)[\mathrm{ORNL}]$

- Midplane tangential bolometer array

- IR cameras

\section{Fast Particles}

- Fission chamber neutron measurement

- Fast neutron measurement

- Scanning neutral particle analyzer

- Fast ion loss scintillator probe*

\section{Edge/Divertor}

- Fast scanning edge probe [UCSD]

- Divertor IR camera [Hiroshima]

- Divertor bolometer*

- Fast pressure gauges [UWash]

- Target Langmuir probes

- Scrape-off layer reflectometer [ORNL]

Table 1. Listed are the existing NSTX diagnostics set and installation plan as of August, 2002. '*' indicates diagnostic is currently being installed. '"' indicates installation planned in the next 1-2 years. Collaborating institutions are indicated in square brackets. 


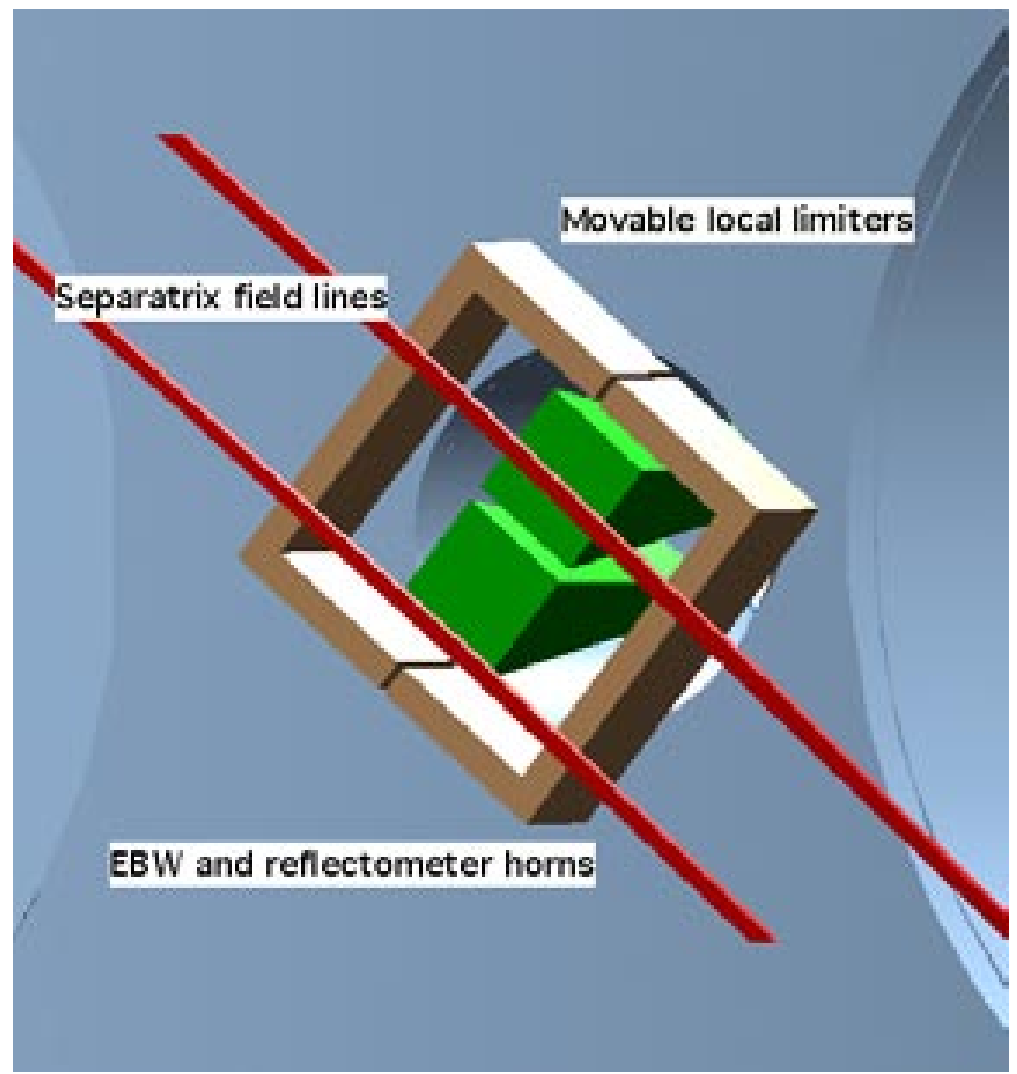

Figure 2. In-vessel schematic view of EBW receiver configuration, showing the orientation of a pair of independently movable local limiters and the EBW and reflectometer horns relative to the edge magnetic field at the outer midplane. 


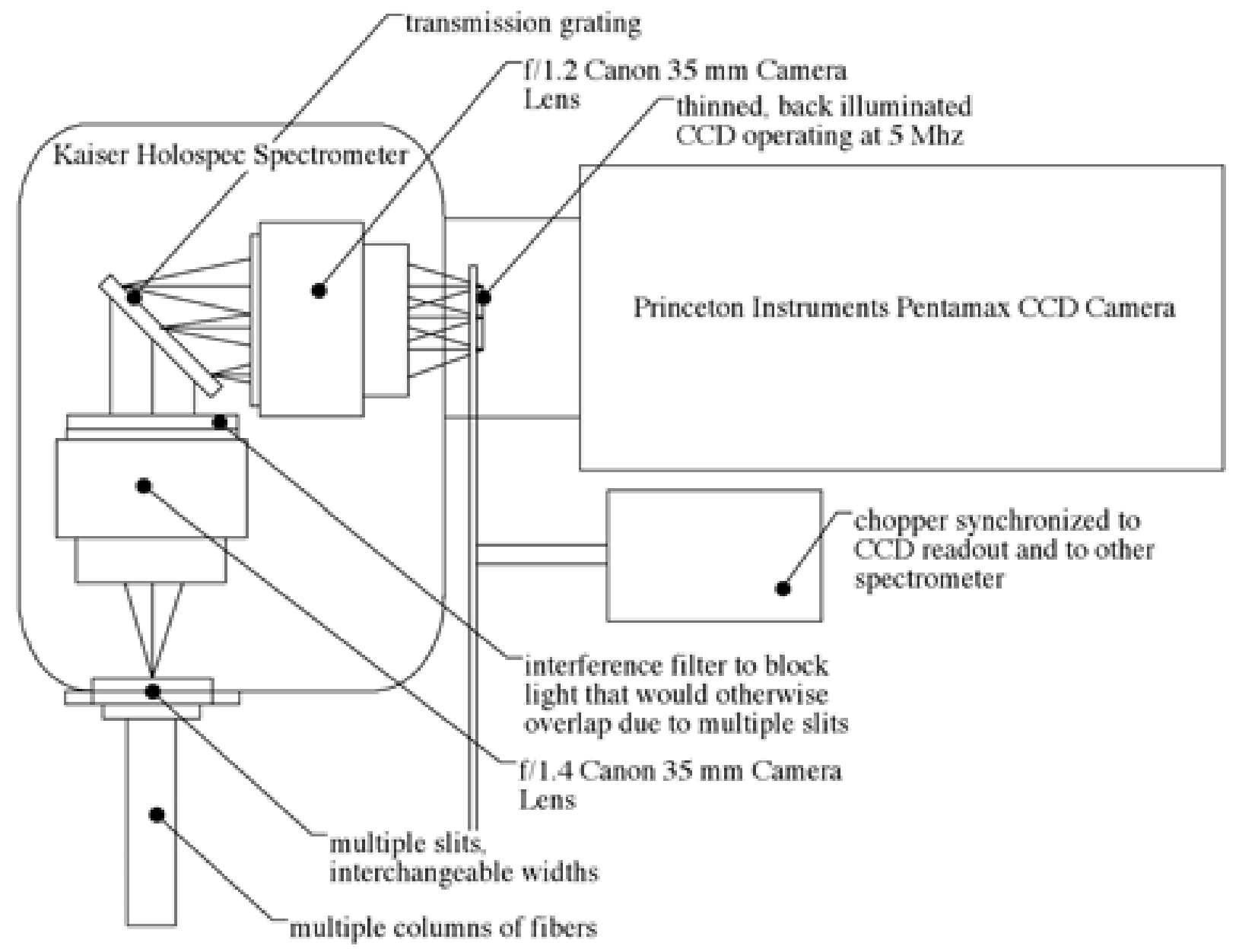

Figure 3. Top schematic view of spectrometer/detector configuration used in CHERS and edge rotation diagnostics. Actual size is $\sim 30 \times 50 \mathrm{~cm}$. 


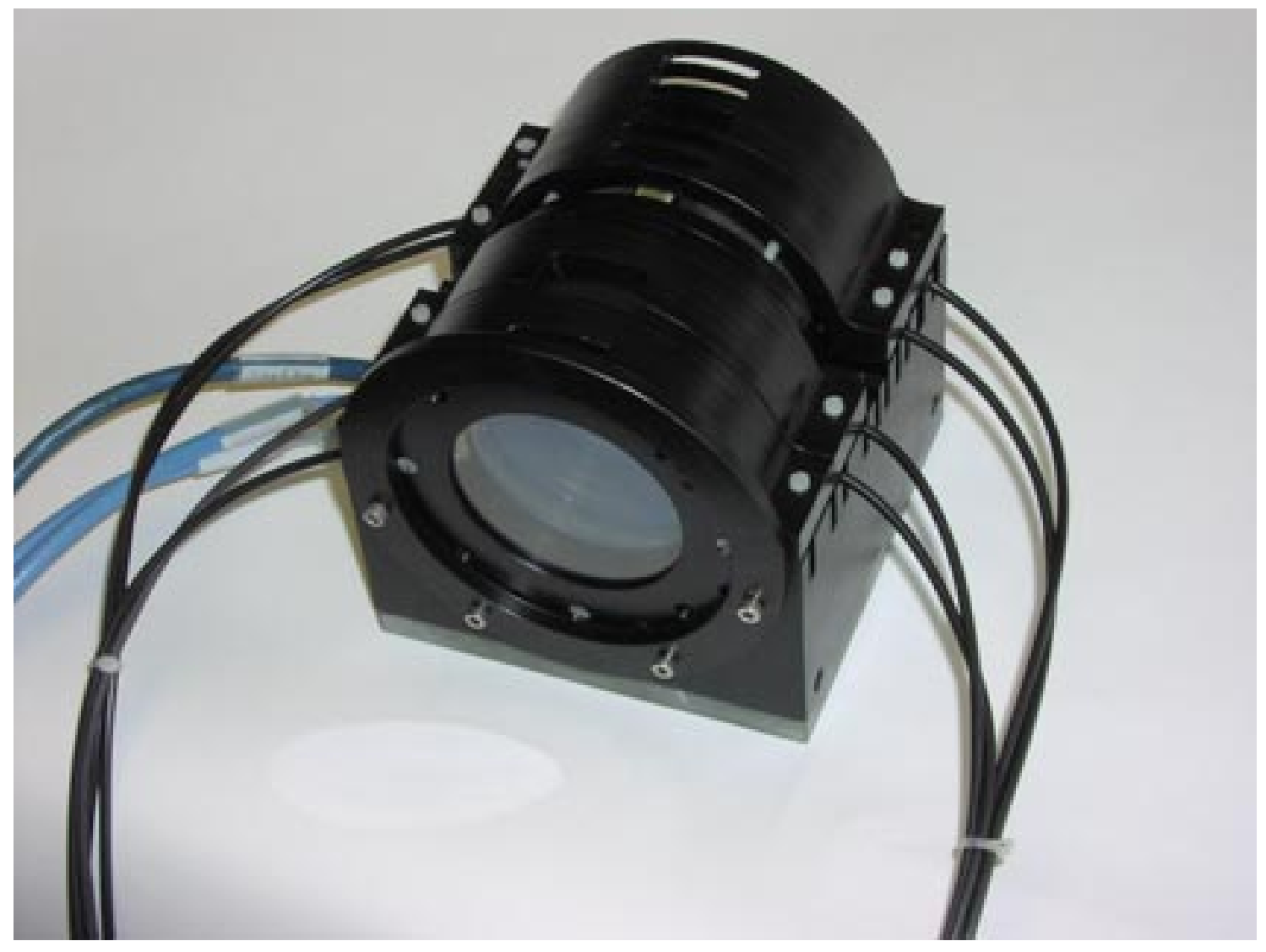

Figure 4. Photograph of 3 stage Lyot filter developed by Nova Photonics for the NSTX MSE/CIF diagnostic. Filter features electronic tuning. 

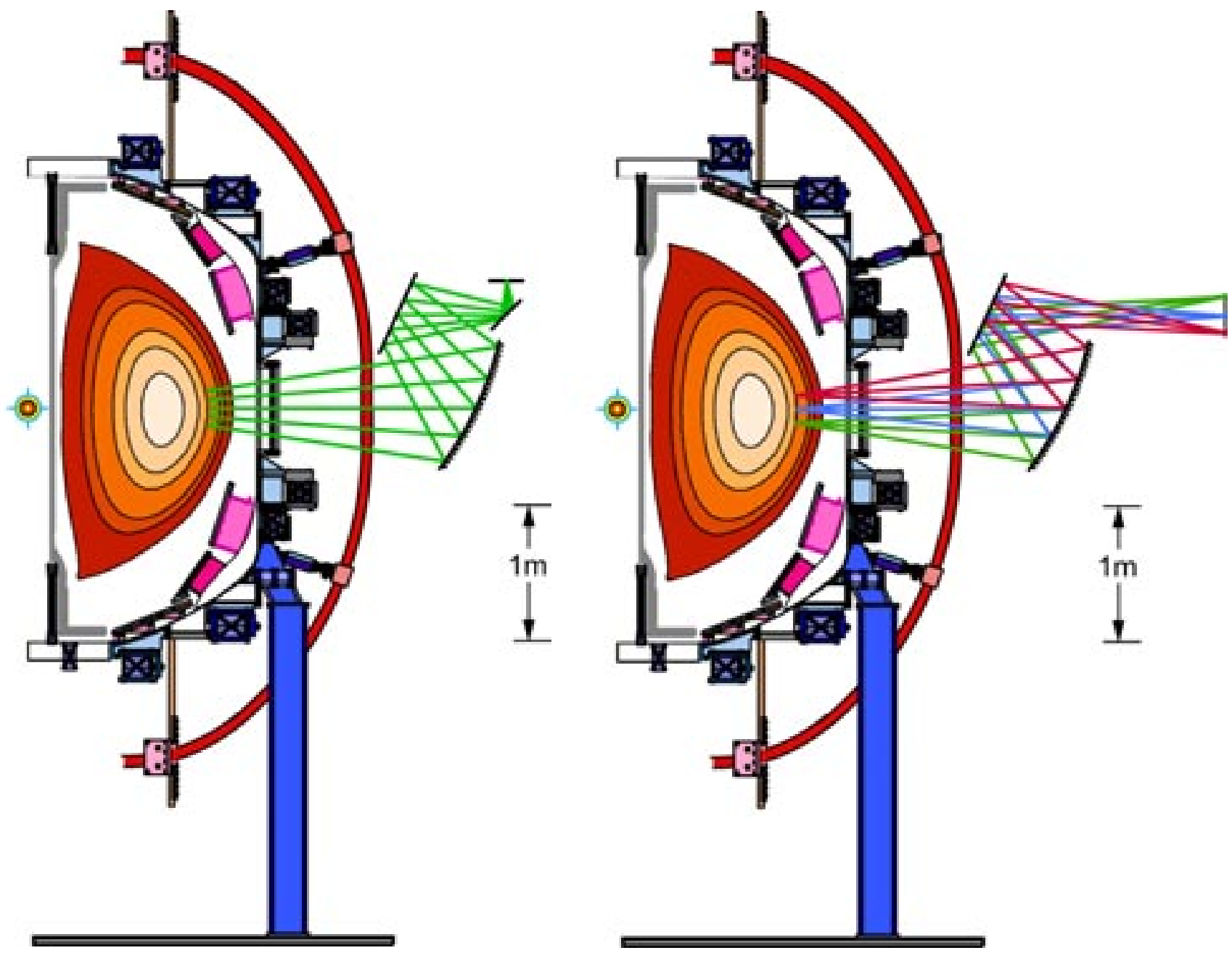

Figure 5. Optical design of illumination and detection optics for proposed microwave imaging reflectometer system on NSTX. 


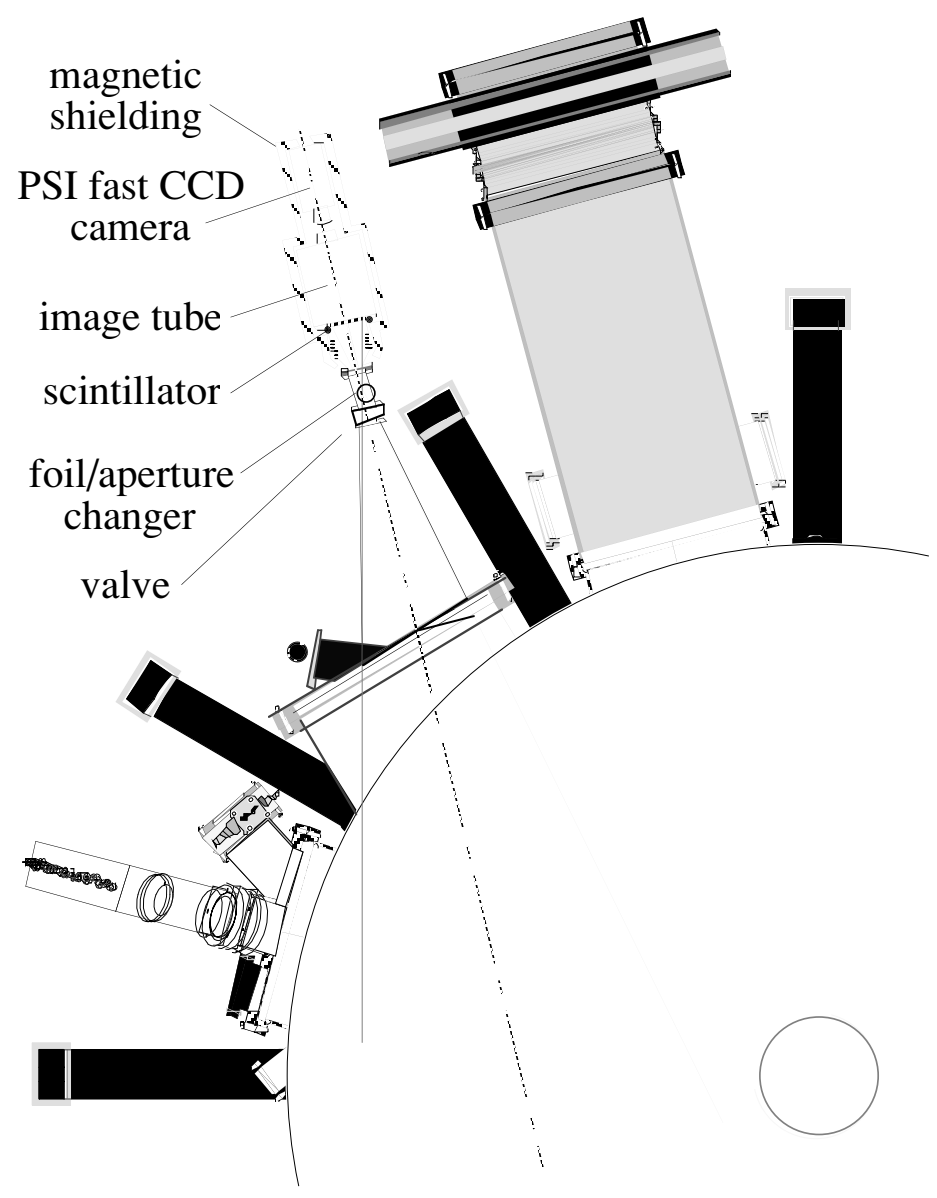

Figure 6. Top view of burst-mode fast tangential x-ray camera. 


\section{External Distribution}

Plasma Research Laboratory, Australian National University, Australia

Professor I.R. Jones, Flinders University, Australia

Professor João Canalle, Instituto de Fisica DEQ/IF - UERJ, Brazil

Mr. Gerson O. Ludwig, Instituto Nacional de Pesquisas, Brazil

Dr. P.H. Sakanaka, Instituto Fisica, Brazil

The Librarian, Culham Laboratory, England

Mrs. S.A. Hutchinson, JET Library, England

Professor M.N. Bussac, Ecole Polytechnique, France

Librarian, Max-Planck-Institut für Plasmaphysik, Germany

Jolan Moldvai, Reports Library, MTA KFKI-ATKI, Hungary

Dr. P. Kaw, Institute for Plasma Research, India

Ms. P.J. Pathak, Librarian, Insitute for Plasma Research, India

Ms. Clelia De Palo, Associazione EURATOM-ENEA, Italy

Dr. G. Grosso, Instituto di Fisica del Plasma, Italy

Librarian, Naka Fusion Research Establishment, JAERI, Japan

Library, Plasma Physics Laboratory, Kyoto University, Japan

Research Information Center, National Institute for Fusion Science, Japan

Dr. O. Mitarai, Kyushu Tokai University, Japan

Dr. Jiangang Li, Institute of Plasma Physics, Chinese Academy of Sciences, People's Republic of China

Professor Yuping Huo, School of Physical Science and Technology, People's Republic of China

Library, Academia Sinica, Institute of Plasma Physics, People's Republic of China

Librarian, Institute of Physics, Chinese Academy of Sciences, People's Republic of China

Dr. S. Mirnov, TRINITI, Troitsk, Russian Federation, Russia

Dr. V.S. Strelkov, Kurchatov Institute, Russian Federation, Russia

Professor Peter Lukac, Katedra Fyziky Plazmy MFF UK, Mlynska dolina F-2, Komenskeho Univerzita, SK-842 15 Bratislava, Slovakia

Dr. G.S. Lee, Korea Basic Science Institute, South Korea

Institute for Plasma Research, University of Maryland, USA

Librarian, Fusion Energy Division, Oak Ridge National Laboratory, USA

Librarian, Institute of Fusion Studies, University of Texas, USA

Librarian, Magnetic Fusion Program, Lawrence Livermore National Laboratory, USA

Library, General Atomics, USA

Plasma Physics Group, Fusion Energy Research Program, University of California at San Diego, USA

Plasma Physics Library, Columbia University, USA

Alkesh Punjabi, Center for Fusion Research and Training, Hampton University, USA

Dr. W.M. Stacey, Fusion Research Center, Georgia Institute of Technology, USA

Dr. John Willis, U.S. Department of Energy, Office of Fusion Energy Sciences, USA

Mr. Paul H. Wright, Indianapolis, Indiana, USA 
The Princeton Plasma Physics Laboratory is operated by Princeton University under contract with the U.S. Department of Energy.

\author{
Information Services \\ Princeton Plasma Physics Laboratory \\ P.O. Box 451 \\ Princeton, NJ 08543
}

Phone: 609-243-2750

Fax: 609-243-2751

e-mail: pppl_info@pppl.gov

Internet Address: http://www.pppl.gov 